\title{
PLAQUE FORMATION AT THE LEFT CORONARY ARTERY: ANALYSIS OF THE RELATIONSHIP BETWEEN ARTERIAL ANGULATIONS AND HEMODYNAMICS
}

\author{
Thanapong Chaichana and Zhonghua Sun \\ Department of Imaging and Applied Physics, \\ Curtin University, \\ Perth, Western Australia, 6102, Australia. \\ 14132256@student.curtin.edu.au,z.sun@curtin.edu.au
}

\begin{abstract}
We investigate the relationship between coronary angulations and their hemodynamic effect. Six left coronary models were generated based on the anatomical details of patient specific data. Varying angles at $90^{\circ}, 75^{\circ}$, $60^{\circ}, 45^{\circ}, 30^{\circ}$ and $15^{\circ}$ between the left anterior descending and the left circumflex branches are simulated. Numerical simulation is used to analyse flow velocity, wall pressure and wall shear stress under normal physiological conditions of human body in different cardiac cycles. Our results showed that the wall shear stress was significantly decreased in models of higher angulations. In contrast, high wall pressure occurred at coronary bifurcation regions with those models. Our preliminary study demonstrates that there is direct relationship between angulations at the left coronary artery and development of atherosclerosis.
\end{abstract}

\section{KEY WORDS}

Atherosclerosis, Coronary bifurcation, Wall shear stress, Wall pressure, Velocity, Numerical simulation.

\section{Introduction}

Coronary Artery Disease (CAD) is the main cause of death in western countries [1,2]. Early detection and diagnosis of CAD is important for the reduction of mortality and subsequent complications [3]. The most common cause of $\mathrm{CAD}$ is atherosclerosis which is affected by the presence of plaques on the artery wall and resulting in lumen stenosis. Plaques have been particularly involved in blood clot and blocks blood circulation to the myocardium. This occurs when the coronary plaques suddenly rupture. When a clot cannot be treated in time, the heart muscle will be impaired due to ischemic changes, which leads to ischemia or myocardial infarction or necrosis [3].

Atherosclerosis is the primary cause of $\mathrm{CAD}$ and plaques are widely distributed along the coronary arteries. However, the situation of plaques is not equally distributed within the coronary artery compared with the orderly risk factors. This is due to the fact that there are

\author{
Kelvin K. L. Wong and Jiyuan Tu \\ School of Aerospace, Mechanical \& Manufacturing \\ Engineering, and Health Innovations Research Institute \\ (HIRi), RMIT University, Australia. \\ \{K.Wong, Jiyuan.Tu\}@ rmit.edu.au.
}

other local conditions that induce plaque formation and progression $[4,5]$. Studies have shown that plaques tend to form in areas of low shear stress or regions of turbulent flow. According to the coronary anatomy, the plaques most frequently occur in the left coronary bifurcation [6]. Computational fluid dynamic (CFD) allows for efficient and accurate computations of the hemodynamic features of both normal and abnormal conditions in the cardiovascular system, as well as in vivo simulation of coronary artery flow [7]. Medical imaging visualisation as medical imaging techniques such as coronary angiography or computed tomography angiography provide anatomic changes of the coronary artery wall due to presence of plaques, thus assessing the degree of lumen stenosis. In contrast, CFD enables simulation and visualisation of the coronary artery flow, even before the plaques are actually formed in the artery wall. Therefore, to some extent, CFD allows early detection of coronary artery disease and improves understanding of the progression of plaques, which are considered to be of paramount importance to clinical treatment. This study aims to investigate hemodynamic in coronary models with varying angulations. It is expected that different angles in the left coronary bifurcation will provide variable effect on flow in the coronary artery. Therefore, the proposed study will serve the purpose of identifying patients that have the potential risk of developing CAD.

\section{Materials and Methods}

\subsection{Patients data with coronary artery disease}

A total of 20 patients (13 men, 7 women, mean age, 54 years \pm 8 ) that are suspected of CAD undergo multislice CT examinations during the first stage of this study. Multislice computed tomography scans were performed with a 64-slice scanner (GE Medical Systems, Lightspeed VCT, GE Healthcare, Milwaukee, WI, USA). Then, 2D and $3 \mathrm{D}$ images were generated to reconstruct the coronary plaques with respect to the angulation of the left coronary artery. 


\subsection{Information about Left Coronary Artery}

A number of coronary models were generated with different angulations between the left anterior descending and left circumflex. Figure 1 shows the 3D visualisation of a left coronary artery consisting of the main stem, and its two branches. The angles between the LAD and LCx are set at $15^{\circ}, 30^{\circ}, 45^{\circ}, 60^{\circ}, 75^{\circ}$ and $90^{\circ}$, respectively. Thus, a total of 6 models are introduced in this study for analysis. Figure 2 shows the variable angles that were simulated in the left coronary artery models.

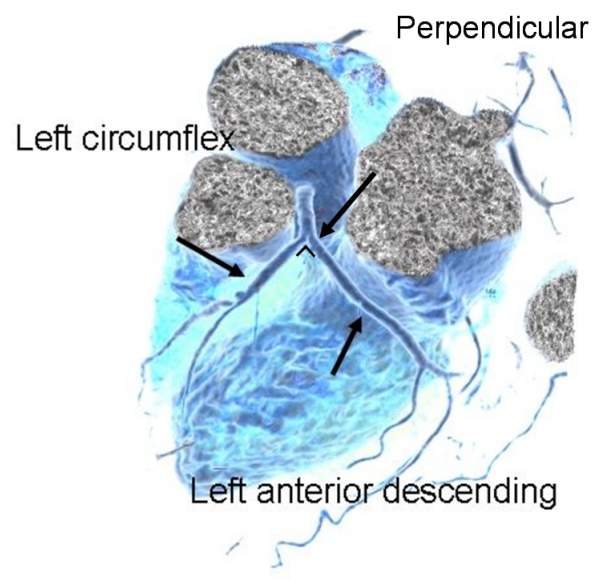

Figure 1. LCA branches on 3D CT visualisation image

Table 1

The measurements of nature LCA model

\begin{tabular}{|l|c|l|c|}
\hline \multicolumn{2}{|l|}{ Diameter of LCA (mm) } & \multicolumn{2}{|c|}{$\begin{array}{l}\text { Distance between bifurcation } \\
\text { to distal/proximal (mm) }\end{array}$} \\
\hline LMS & 3.0 & Proximal LMS & 35 \\
\hline LAD & 2.0 & Distal LAD & 25 \\
\hline LCx & 1.5 & Distal LCx & 20 \\
\hline
\end{tabular}

LMS-left main stem

\subsection{Generation of left coronary Models}

The surface model was generated based on the anatomic details of LCA in Section 2.1 and 2.2 with reference to the LCA anatomy in Figure 1 and the parameters in Table 1. CATIA V5 R18 (Dassault Systemes, Inc., Suresnes, France) is used. The surface model is then converted into the solid model, as shown in Figure 3. According to The $90^{\circ}$ model, other five models were generated by varying the degree of the $90^{\circ}$ model between LCx and LAD, as shown in Figure 2 and all of models are saved in STP mesh format.

\subsection{Generation of Mesh Models}

The 6 computer aided design (CAD) models of left coronary were used for mesh generation and CFD simulation. The CAD models are produced using a tetrameshing and three prism layers method. Details of meshing elements are shown in Table 2. The mesh models were generated with the ANSYS ICEM CFD version 12 (ANSYS, Inc., Canonsburg, PA, USA). Finally, the 6 mesh models were saved in GTM format for CFD computation.

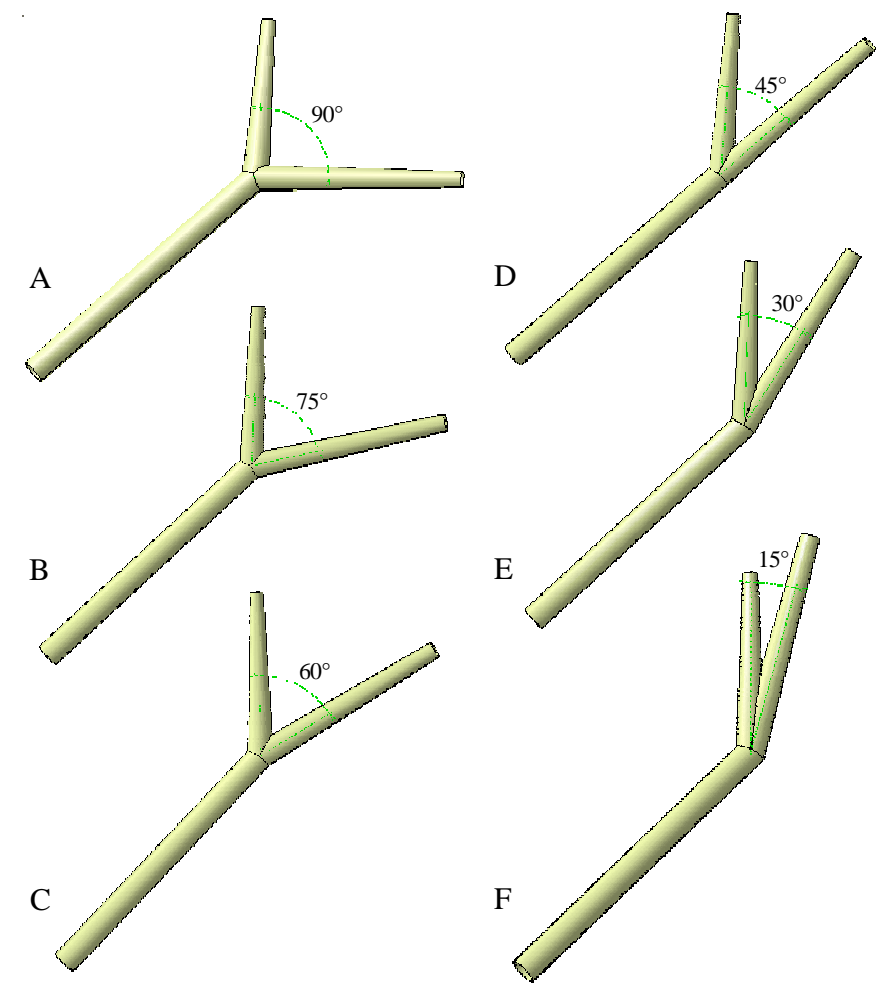

Figure 2. Diagrams showing variable angles of LCA, $90^{\circ}(\mathrm{A}), 75^{\circ}(\mathrm{B}), 60^{\circ}(\mathrm{C}), 45^{\circ}(\mathrm{D}), 30^{\circ}(\mathrm{E})$ and $15^{\circ}(\mathrm{F})$

Table 2

The details of meshing elements

\begin{tabular}{|c|c|c|c|}
\hline \multicolumn{4}{|c|}{ The 6 LCA models } \\
\hline Models & Angles & Tetra-mesh & 3 Prism layers \\
\hline A & $90^{\circ}$ & 354827 & 97677 \\
\hline B & $75^{\circ}$ & 357502 & 99153 \\
\hline C & $60^{\circ}$ & 366925 & 103941 \\
\hline D & $45^{\circ}$ & 364552 & 104106 \\
\hline E & $30^{\circ}$ & 358038 & 98988 \\
\hline F & $15^{\circ}$ & 330427 & 92517 \\
\hline
\end{tabular}




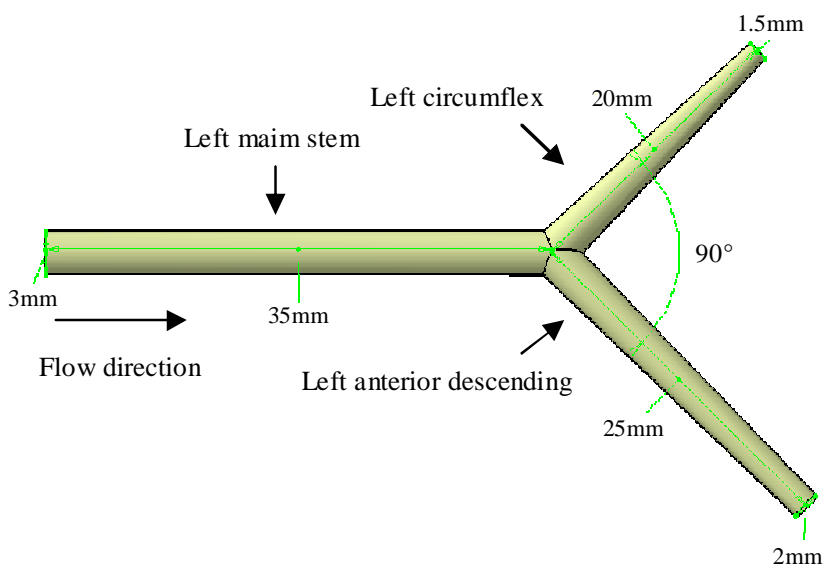

Figure 3. The $90^{\circ}$ model based on patient specific anatomic details

\subsection{CFD simulation in left coronary artery}

\subsubsection{Application of physiological parameters}

In order to ensure that our analysis reflects the realistic simulation of in vivo conditions, application of the physiological parameters should be considered for threedimensional numerical analysis. The transient simulation is performed, and accurate hemodynamic rheological and material properties are used in this study, as referred to in previous studies [8]. According to Bertolotti et al [9], we have reconstructed the flow rate graph at LCA by developing algorithm for creation of Fourier series graph as shown in Figure 4 . The boundary conditions were determined by pulsatile velocity at left main stem (inlet) and opening pressure with left anterior descending (outlet) and left circumflex (outlet). Accurate physiological parameters were applied with a blood density of 1060 $\mathrm{kg} / \mathrm{m}^{3}$, and a blood viscosity of $0.0027 \mathrm{~Pa} \mathrm{~s}$. The blood flow is assumed to be laminar with no-slip conditions, Newtonian [10,11] and incompressible [12].

\subsubsection{Performance of CFD computation}

To ensure that our results are valid, we perform numerical simulation using ANSYS CFX version 12 (ANSYS, Inc.) based on a 4 MB RAM and a dual core $2.4 \mathrm{GHz}$ CPU. The left coronary artery in normal flow situation is governed by Navier-Stokes equations. The CFD solution was fully converged using 32 time steps, with each 0.05 step within 1.6 second of the pulsatile velocity flow. The computed time consumption for each LCA model was approximately one and half hour. This computation was performed similar to an in vivo condition by referring to previous experiments $[13,14,15]$. The flow pattern, flow velocity, wall pressure, and wall shear stress were calculated and measured in this study.

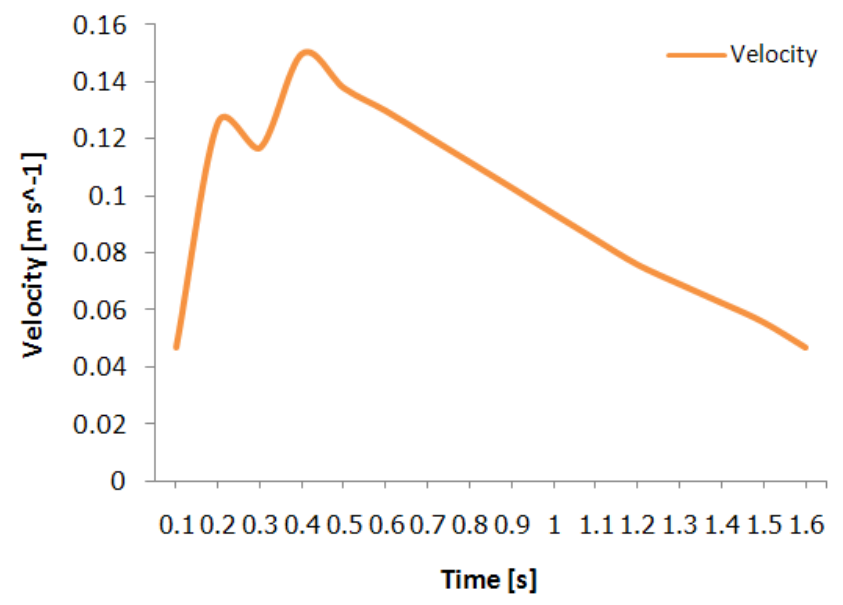

Figure 4. Pulsatile velocity at left coronary artery

\section{Results}

Of the 19 patients with left anterior descending (LAD) disease, $74 \%$ had a bifurcation angle $>80^{\circ}$, and $88 \%$ of the patients with diseased left circumflex (LCx) had a bifurcation angle $>80^{\circ}$, whereas $88 \%$ of the patients with both left anterior descending and circumflex disease had a bifurcation angle $>90^{\circ}$. This indicates a strong correlation between wider angulation and formation of coronary plaques.

CFD simulation is performed for six coronary models under realistic physiological condition during systolic and diastolic phases. Our results showed a direct relationship between CFD analysis and the simulated angulations in these models. Peak velocity reached the highest during systolic at $0.4 \mathrm{sec}$ (Figure 4). A laminar flow was noticed at small angled models, and it became turbulent at wide angled models, as shown in Figure 5.

The wall shear stress (WSS) is found to decrease in wide angled models when compared to narrowed models. This is especially obvious in the systolic phase as shown in Figure 6. Low WSS occurs at the bifurcation location where the left coronary main stem branches into $\mathrm{LAD}$ and LCx.

The wall pressure also changes with the variation of angulations in different models. Wall pressure decreased from widely angled models to narrowly angled models, as shown in Figure 7. This is especially apparent in the model with a $15^{\circ}$ angulation as the results showed that the wall pressure reduced significantly when the blood flows through from the left main stem to the LAD and $\mathrm{LCx}$ braches. 


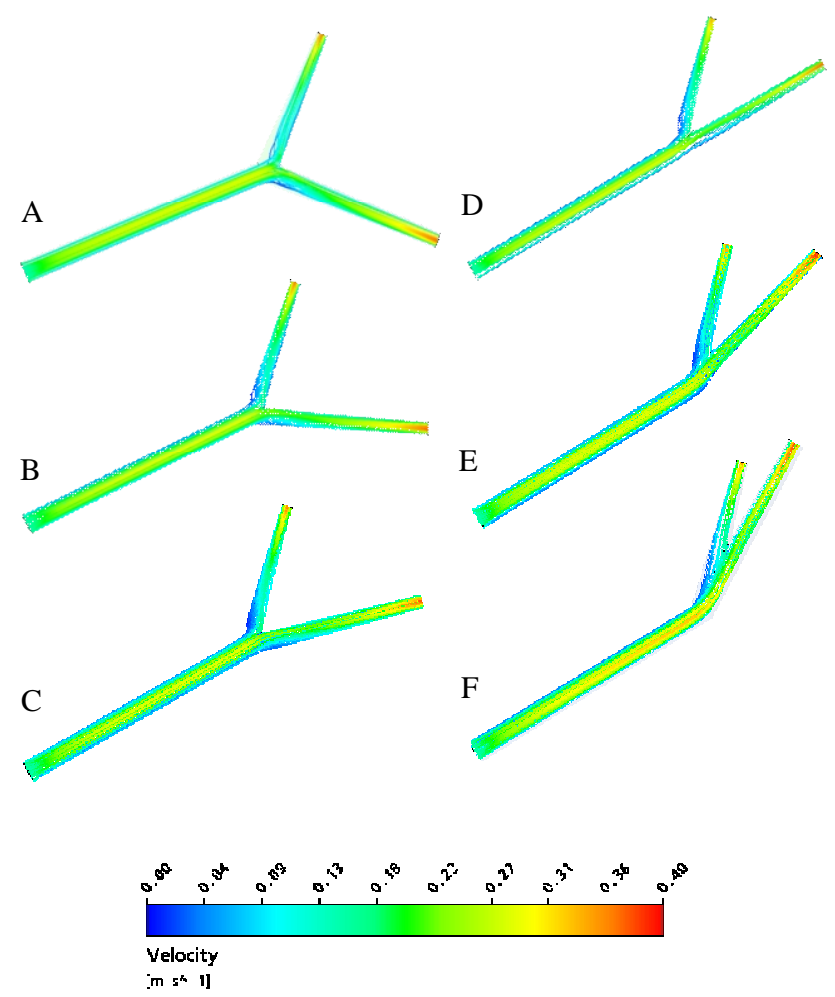

Figure 5. Velocity showing variable angles of LCA, A-F: measured at systolic peak $0.4 \mathrm{sec}$

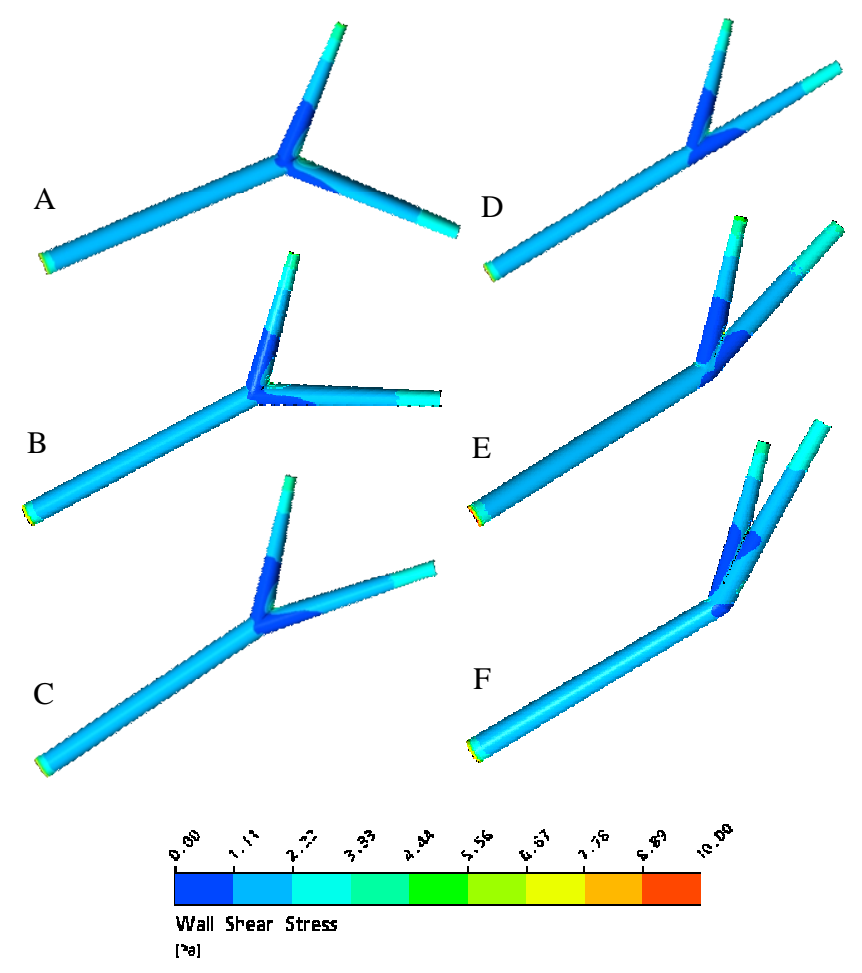

Figure 6. Wall shear stress with variable angles at $90^{\circ}$
(A), $75^{\circ}(\mathrm{B}), 60^{\circ}(\mathrm{C}), 45^{\circ}(\mathrm{D}), 30^{\circ}(\mathrm{E})$ and $15^{\circ}(\mathrm{F})$

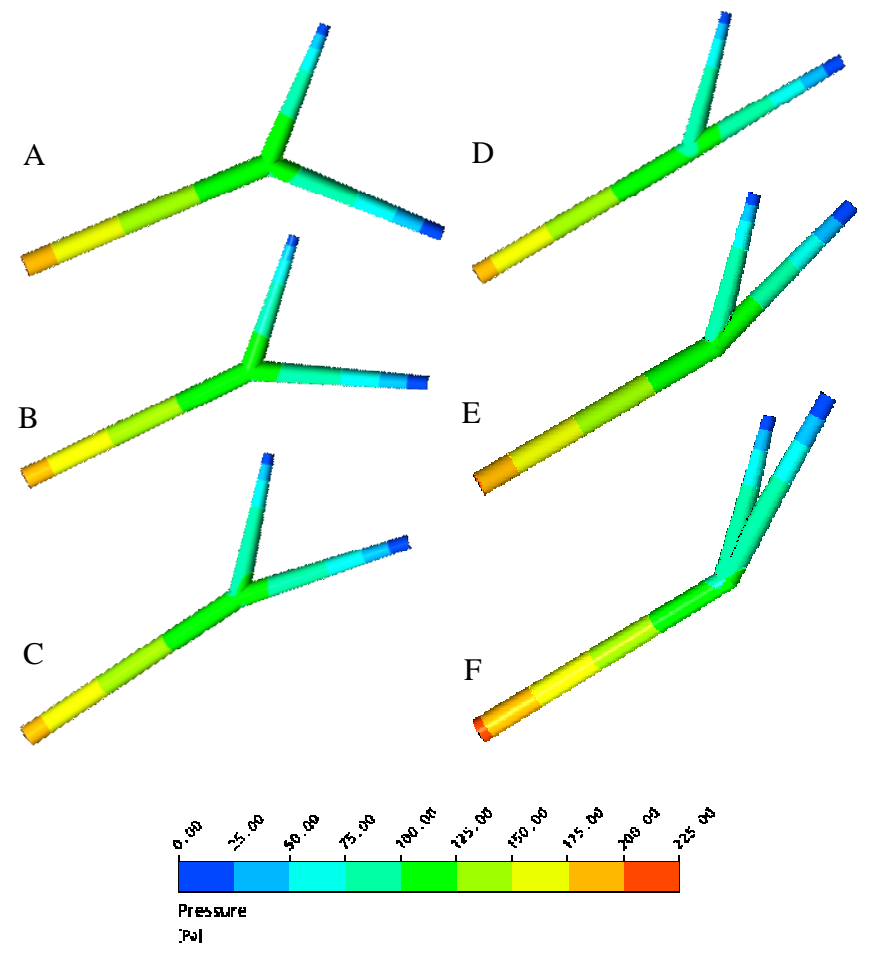

Figure 7. Wall pressure with variable angles at $90^{\circ}(\mathrm{A})$, $75^{\circ}(\mathrm{B}), 60^{\circ}(\mathrm{C}), 45^{\circ}(\mathrm{D}), 30^{\circ}(\mathrm{E})$ and $15^{\circ}(\mathrm{F})$

\section{Discussion and Conclusion}

We have simulated the hemodynamic features in a number of coronary models with different angles at the left bifurcation, and correlated the CFD analysis with patient specific data. Our results showed that the wall shear stress decreased significantly with wide angulations in the left coronary bifurcation, and this leads to the formation of atherosclerosis.

Curvatures and bifurcation of large and medium sized arteries are severely affected by atherosclerosis such as abdominal aorta and carotid artery disease. Numerous studies point out that there is a relationship between the genesis and the progression of the disease with the locally irregular low field occurrence in these zones. This also applies to smaller sized arteries such as coronary artery. This is consistent with previous studies [16-18].

Obviously, the blood flow velocity was noticed to be laminar at models with smaller angles whereas the flow velocity became turbulent at widely angled models (Figure 5). Flow patterns in the arterial tree are changed considerably by alterations of pulsatility. Although we did not take into consideration of the pulsatility of the artery wall, our results are valid and could be used for further studies to improve understanding of the atherosclerosis at the coronary artery. 
The limitation of this study is its lack of realistic modeling, and thus in the future study, we will perform computation fluid dynamic analysis in the coronary models from patient specific data (based on coronary remodelling based on CT scan data), and with different degree of coronary stenosis and locations of coronary plaques (19).

In conclusion, we have performed CFD analysis to examine the hemodynamics of coronary models with different angles at the left bifurcation. Our preliminary study shows a direct relationship between arterial angulations and low shear stress, leading to formation of atherosclerosis.

\section{Acknowledgement}

The PhD scholarship awarded by E-Medicine centre, Western Australia is greatly appreciated.

\section{References}

[1] American Heart Association, 2002 Heart and Stroke Statistical Update (Dallas, Tex.: American Heart Association, 2001).

[2] American Heart Association, Heart Disease and Stroke Statistics-2008 Update, American Heart Association. Statistical Fact Sheets. Available at: http://circ.ahajournals.org/cgi/content/full/117/4/e25.

Accessed September 28, 2010.

[3] Australian Institute of Health and Welfare, The tenth biennial health report of the Australian Institute of Health and Welfare, Cat. no. AUS 73. Canberra: AIHW, 2006.

[4] Sabbah HN, Khaja F, Hawkins ET, Brymer JF, McFarland TM, Bel-Kahn J, Doerger PT, Stein PD, 1986, "Relation of atherosclerosis to arterial wall shear in the left anterior descending coronary artery of man", American Heart Journal, vol. 112, pp. 453-458.

[5] Jeremias A, Huegel H, Lee DP, Hassan A, Wolf A, Yeung AC, Yock PG, Fitzgerald PJ, 2000, "Spatial orientation of atherosclerotic plaque in non-branching coronary artery segments", Atherosclerosis, vol. 152, pp. 209-215.

[6] Bruschke AV, Wijers TS, Kolsters W, Landmann J, 1981, "The anatomic evolution of coronary artery disease Demonstrated by coronary arteriography in 256 nonoperated patients", Circulation, vol. 63, pp. 527-536.

[7] Shanmugavelayudam S, Rubenstein D and Yin W, 2010, "Effect of geometrical assumptions on numerical modelling of coronary blood flow under normal and disease conditions" Journal of Biomechanical Engineering, vol. 132, pp. 1-8.

[8] Frauenfelder T, Lotfey M, Boehm T and Wildermuth S, 2006, "Computational fluid dynamics: hemodynamic changes in abdominal aortic aneurysm after stent-graft implantation", CardioVascular and Intervenlional, vol. 29, no. 4 , pp. 724 .

[9] Bertolotti C, Deplanoa V, Fuseri J and Dupouyb P, 2001, "Numerical and experimental models of postoperative realistic flows in stenosed coronary bypasses", Journal of Biomechanics, vol. 34, pp. 1049-1064.

[10] Johnston B, Johnston P, Corney S and Kilpatrick D, 2006, "Non-Newtonian blood flow in human right coronary arteries: Transient simulations", Journal of Biomechanics, vol. 39, pp. 1116-1128.

[11] Johnston B, Johnston P, Corney S and Kilpatrick D, 2004, "Non-Newtonian blood flow in human right coronary arteries: steady state simulations", Journal of Biomechanics, vol. 37, pp. 709.

[12] Borgh A, Wood N, Mohiaddin R, and Xu X, 2008, "Fluid-solid interaction simulation of flow and stress pattern in thoracoabdominal aneurysms: A patientspecific study", Journal of Fluids and Structures, vol. 24, no. 2, pp. 270-28.

[13] Sun Z and Chaichana T, 2010, "Fenestrated Stent Graft Repair of Abdominal Aortic Aneurysm: Hemodynamic Analysis of the Effect of Fenestrated Stents on the Renal Arteries", Korean Journal Radiology, vol. 11, no. 1, pp. 95-106.

[14] Sun Z and Chaichana T, 2009, "Investigation of the Hemodynamic Effect of Stent Wires on Renal Arteries in Patients with Abdominal Aortic Aneurysms Treated with Suprarenal Stent-Grafts", CardioVascular and Interventional Radiology, vol. 32, pp. 647-657.

[15] Sun Z, Chaichana T, Mwipatayi B and Ng C, 2009, "Hemodynamic effect of calcified plaque on blood flow in carotid artery disease: A preliminary study", Proceedings of the 3rd International conference on Bioinformatics and Biomedical Engineering, Beijing, pp. $1-4$.

[16] Ribicki F, Melchionna S, Mitsouras D, Coskun A, Whitmore A, Steigner M, Nallamshetty L, Welt F, Bernaschi M, Borkin M, Sircar J, Kaxiras E, Succi S, Stone P and Feldman C, 2009, "Prediction of coronary artery plaque progression and potential rupture from 320detector row prospectively ECG-gated single heart beat CT angiography: Lattice Boltzmann evaluation of endothelial shear stress", International Journal of Cardiovascular Imaging, vol. 25. Pp. 289-299.

[17] Soulis V, Farmakis T, Giannoglou G and Louridas G, 2006, "Wall shear stress in normal left coronary artery tree", Journal of Biomechanics, vol. 39, pp. 742-743.

[18] Chatzizisis YS, Coskum AU, Jonas M, Edelman ER, Feldman CL and Stone PH, 2007, "Role of endothelial shear stress in the natural history of coronary atherosclerosis and vascular remodelling", Journal of the American College of Cardiology, vol. 49, pp. 2379.

[19] Katritsis G, Theodorakakos A, Pantos I, Andriotis A, Efstathopoulos E, Siontis G, Karcanias N, Redwood S and Gavaises M, "Vortex formation and recirculation zones in left anterior descending artery stenoses: computational fluid dynamic analysis", Physics in Medicine and Biology, vol 55, pp. 1395-1411, 2010. 